\title{
Multivalent Neoglycoconjugates by Regiospecific Cycloaddition of Alkynes and Azides Using Organic-Soluble Copper Catalysts
}

\author{
Francisco Pérez-Balderas, Mariano Ortega-Muñoz, Julia Morales-Sanfrutos, Fernando \\ Hernández-Mateo, Francisco G. Calvo-Flores, Jose A. Calvo-Asín, Joaquín Isac-García, \\ Francisco Santoyo-González*
}

Instituto de Biotecnología, Facultad de Ciencias, Universidad de Granada, E-18071 Granada, Spain

\section{SUPPORTING INFORMATION}

\section{General Experimental}

TLC was performed on Merck Silica Gel $60 \mathrm{~F}_{254}$ aluminium sheets. Reagent used for developing plates include ceric sulphate $(1 \% \mathrm{w} / \mathrm{v})$ and ammonium sulphate $(2.5 \% \mathrm{w} / \mathrm{v})$ in $10 \%$ (v/v) aqueous sulphuric acid, iodine, ethanolic sulfuric acid (10\% v/v) and by UV light when applicable. Flash column chromatography was performed on Silica Gel Scharlau (230-400 mesh, ASTM). Melting points were measured on a Gallenkamp melting point apparatus and are uncorrected. Optical rotations were recorded on a Perkin-Elmer 141 polarimeter at room temperature. IR spectra were recorded on a Satellite Mattson FTIR. ${ }^{1} \mathrm{H}$ and ${ }^{13} \mathrm{C}$ NMR spectra were recorded at room temperature on a Bruker (300-400 MHz) spectrometer. Chemical shifts are given in ppm and referenced to internal $\mathrm{CDCl}_{3} . J$ values are given in $\mathrm{Hz}$. FAB mass spectra were recorded on a Fissons VG Autospec-Q spectrometer, using m-nitrobenzyl alcohol or thioglycerol as matrix

\section{Starting materials}

Propargyl amine, $\alpha, \alpha^{\prime}$-dichloro- $m$-xilene, cyanuric chloride, and 1,2,4,5tetrakis(bromomethyl)benzene were purchased from Aldrich. Compound $\mathbf{1 a},{ }^{1,2} \mathbf{1 b},{ }^{3} \mathbf{2 a},{ }^{4} \mathbf{6 d},{ }^{5}$ $\mathbf{6 b},{ }^{6} \mathbf{6 c},,^{6,7}$ and $\mathbf{6} \mathbf{e}^{8 ; 9}$ were obtained following the procedures described in literature. Compound 4b was obtained following a similar procedure as described ${ }^{10}$ in $98 \%$ yield. Compound $\mathbf{6 c}$ was obtained from hexakis(bromomethyl)benzene. ${ }^{11}$

N-Acetyl-N-prop-2-ynyl 2,3,4,6-tetra- $O$-acetyl- $\alpha$-D-mannopyranosylamine (1c). The title compound was obtained following the procedure described for the synthesis of analogous mannopyranosylamines. ${ }^{12}$ A mixture of mannose $(2.1 \mathrm{~g}, 11.6 \mathrm{mmol})$, propargylamine $(0.66 \mathrm{~g}, 12 \mathrm{mmol})$ in methanol $(7 \mathrm{~mL})$ was stirred at room temperature under a nitrogen 
atmosphere for 2 days. Evaporation of the solvent was followed by conventional acetylation with $\mathrm{Ac}_{2} \mathrm{O}-\mathrm{Py}(15: 10 \mathrm{~mL})$ giving a crude product that was purified by column chromatography (ether) yielding $1 \mathrm{~g}(2.53 \mathrm{~g}, 51 \%)$ as a foam: $[\alpha]_{\mathrm{D}}+47^{\circ}(c 1$, chloroform); IR $(\mathrm{KBr})$ : 3482,3280 , 1752, 1670, 1226, $1054 \mathrm{~cm}^{-1}$; $1 \mathrm{H} \mathrm{NMR}\left(\mathrm{CDCl}_{3}, 300 \mathrm{MHz}\right): \delta 5.93$ (s, $\left.1 \mathrm{H}, \mathrm{H}-1\right), 5.48$ (br s, $1 \mathrm{H}$, H-2), 5.22 (t, $1 \mathrm{H}, J=10.0 \mathrm{~Hz}, \mathrm{H}-4), 5.15$ (dd, $1 \mathrm{H}, J=10.0$ and $2.9 \mathrm{~Hz}, \mathrm{H}-3$ ), 4.31 (br d, $1 \mathrm{H}, J$ $\left.=19.2 \mathrm{~Hz}, \mathrm{CH}_{2} \mathrm{~N}\right), 4.26(\mathrm{dd}, 1 \mathrm{H}, J=12.3$ and $5.7 \mathrm{~Hz}, \mathrm{H}-6), 4.14(\mathrm{dd}, 1 \mathrm{H}, J=12.3$ and $2.4 \mathrm{~Hz}$, H-6'), 4.00 (br d, $1 \mathrm{H}, J=19.1, \mathrm{CH}_{2} \mathrm{~N}$ ), 3.81 (ddd, $1 \mathrm{H}, J=10.0,5.8$ and 2.4 Hz, H-5), 2.26 (b $\mathrm{s}, 1 \mathrm{H}, \mathrm{C} \equiv \mathrm{CH}), 2.25,2.21,2.10,2.06,1.96(5 \mathrm{~s}, 15 \mathrm{H}, 5 \mathrm{Ac}) ;{ }^{13} \mathrm{C} \mathrm{NMR}\left(\mathrm{CDCl}_{3}, 75 \mathrm{MHz}\right)$ : $\delta$ 170.6, 169.9, 164.8 (5 CO), $78.3(\mathrm{C}-1), 75.2(\mathrm{C}-5), 72.1(\mathrm{C} \equiv \mathrm{CH}), 71.6(\mathrm{C}-3, \mathrm{C} \equiv C \mathrm{H}), 70.0(\mathrm{C}-$ 2), $65.6(\mathrm{C}-4), 62.6(\mathrm{C}-6), 33.6\left(\mathrm{CH}_{2} \mathrm{~N}\right), 21.9,21.5,20.8,20.6$ (5 Me $\left.\mathrm{CO}\right)$.; HMRS (FAB+) $\mathrm{C}_{19} \mathrm{H}_{25} \mathrm{NO}_{10} \mathrm{Na} 450.1366$. Found 450.1365 .

2,4,6-Tris(2-azidoethoxy)-1,3,5-triazine (6a). To a cold solution of cyanuric chloride $(1.87 \mathrm{~g}, 10 \mathrm{mmol})$ and 2-azido ethanol $(5.2 \mathrm{~g}, 60 \mathrm{mmol})$ in dry acetonitrile $(25 \mathrm{~mL})$ was added dropwise a solution of DIPEA $(7 \mathrm{~mL})$ in acetonitrile $(10 \mathrm{~mL})$. The reaction mixture was kept at room temperature for 2 days. After evaporation the crude product was dissolved in chloroform $(150 \mathrm{~mL})$ and washed with water $(50 \mathrm{~mL})$. The organic phase was dried $\left(\mathrm{Na}_{2} \mathrm{SO}_{4}\right)$, filtered and evaporated. Column chromatography $\left(\mathrm{CH}_{2} \mathrm{Cl}_{2}\right.$-ether $\left.20: 1\right)$ gave $6 \mathbf{a}(2.34 \mathrm{~g}, 70 \%)$ as a liquid. IR (film): 2105, 1554, 1329, $1294 \mathrm{~cm}^{-1}$; ${ }^{1} \mathrm{H} \mathrm{NMR}\left(\mathrm{CDCl}_{3}, 300 \mathrm{MHz}\right): \delta 4.59$ (t, $1 \mathrm{H}, J=5.1 \mathrm{~Hz}$, $\left.\mathrm{CH}_{2} \mathrm{O}\right), 3.68\left(\mathrm{t}, 6 \mathrm{H}, J=5.1 \mathrm{~Hz}, \mathrm{CH}_{2} \mathrm{~N}_{3}\right) ;{ }^{13} \mathrm{C} \mathrm{NMR}\left(\mathrm{CDCl}_{3}, 75 \mathrm{MHz}\right): \delta 171.8,67.3,49.4$.

\section{Cycloaddition compounds}

1-(2',3',4',6',-Tetra-O-acetyl- $\alpha$-D-mannopyranosyloxyethyl)-4-(2',3",,4",6"-tetra$O$-acetyl- $\alpha-D$-mannopyranosyloxymethyl)-1H-[1,2,3]-triazol (3a) (Table 1, entry 1). Foam solid. $[\alpha]_{\mathrm{D}}+37.5^{\circ}$ (c 1, chloroform); IR (film) 1750, 1436, 1369, $1234 \mathrm{~cm}^{-1} ;{ }^{1} \mathrm{H} \mathrm{NMR}\left(\mathrm{CDCl}_{3}\right.$, $300 \mathrm{MHz}$ ): $\delta 7.79$ (s, $1 \mathrm{H}, \mathrm{H}-5), 5.35-5.18$ (m, 6 H, H-2',3',4' ,2",3",4"), 5.00 (s, $1 \mathrm{H}, \mathrm{H}-1$ ), 4.88 $\left(\mathrm{d}, 1 \mathrm{H}, J=12.2 \mathrm{~Hz}, \mathrm{CH}_{2} \mathrm{O}\right), 4.71\left(\mathrm{~d}, 1 \mathrm{H}, J=12.3 \mathrm{~Hz}, \mathrm{CH}_{2} \mathrm{O}\right), 4.82(\mathrm{~s}, 1 \mathrm{H}, \mathrm{H}-1), 4.64(\mathrm{t}, 2 \mathrm{H}$, $\left.J=5.0 \mathrm{~Hz}, \mathrm{CH}_{2} \mathrm{~N}\right), 4.32(\mathrm{dd}, 1 \mathrm{H}, J=12.5$ and $5.1 \mathrm{~Hz}, \mathrm{H}-6), 4.21(\mathrm{dd}, 1 \mathrm{H}, J=12.2$ and 5.1 $\mathrm{Hz}, \mathrm{H}-6$ ), 4.16-4.10 (m, $3 \mathrm{H}, \mathrm{H}-5,5,6), 4.06$ (dd, $1 \mathrm{H}, J=12.4$ and $2.1 \mathrm{~Hz}, \mathrm{H}-6$ ), 3.91 (dt, $1 \mathrm{H}, J$ $=10.5$ and $\left.5.0 \mathrm{~Hz}, \mathrm{OCH}_{2} \mathrm{CH}_{2} \mathrm{~N}\right), 3.59\left(\mathrm{~m}, 1 \mathrm{H}, \mathrm{OCH}_{2} \mathrm{CH}_{2} \mathrm{~N}\right), 2.15,2.14,2.12,2.10,2.05,2.04$, 2.00, 1.97 (8 s, $24 \mathrm{H}, 8 \mathrm{x} \mathrm{Ac}) ;{ }^{13} \mathrm{C} \mathrm{NMR}\left(\mathrm{CDCl}_{3}, 75 \mathrm{MHz}\right): \delta 170.5,170.3,169.8,169.6,169.5$, 169.4, 143.5, 124.3, 97.2, 96.9, 69.3, 68.9, 68.6, 68.5, 66.0, 65.6, 62.2, 62.1, 60.7, 49.6, 24.8, 20.7, 20.6, 20.5; HMRS (FAB+) Calc for $\mathrm{C}_{33} \mathrm{H}_{45} \mathrm{~N}_{3} \mathrm{O}_{20} \mathrm{Na}$ 826.2494. Found 826.2491.

1-(2',3',4',6',-Tetra-O-acetyl- $\alpha$-D-mannopyranosyloxyethyl)-4-(2",3",4",6",-tetra$O$-acetyl- $\alpha$-D-mannopyranosylthiomethyl)-1H-[1,2,3]-triazol (3b) (Table 1, entry 2).. Solid. 
M.p. $63-65^{\circ} \mathrm{C} ;[\alpha]_{\mathrm{D}}+96^{\circ}$ (c 1.16, chloroform); IR (KBr): 1751, 1228, $1050 \mathrm{~cm}^{-1} \mathrm{~cm}^{-1} ;{ }^{1} \mathrm{H}$ NMR $\left(\mathrm{CDCl}_{3}, 300 \mathrm{MHz}\right): \delta 7.64$ (s, $\left.1 \mathrm{H}, \mathrm{H}-5\right), 5.38-5.16$ (m, 7 H, H-1',2',3',4' ,2",3",4"), 4.78 (d, $1 \mathrm{H}$, $\left.J=1.5 \mathrm{~Hz}, \mathrm{H}-1^{\prime}\right), 4.59\left(\mathrm{t}, 2 \mathrm{H}, J=5.3 \mathrm{~Hz}, \mathrm{CH}_{2} \mathrm{~N}\right), 4.40(\mathrm{ddd}, 1 \mathrm{H}, J=9.5,4.9$ and $2.4 \mathrm{~Hz}, \mathrm{H}-$ 5"), 4.31 (dd, $1 \mathrm{H}, J=12.2$ and $4.9 \mathrm{~Hz}, \mathrm{H}-6 "), 4.21$ (dd, $1 \mathrm{H}, J=12.3$ and $5.2 \mathrm{~Hz}, \mathrm{H}-6$ '), 4.154.02 (m, 3 H, H-6',6",CH $2 \mathrm{O}$ ), 3.99 (d, $1 \mathrm{H}, J=14.4 \mathrm{~Hz}, \mathrm{CH}_{2} \mathrm{~S}$ ), 3.88 (d, $1 \mathrm{H}, J=14.5 \mathrm{~Hz}$, $\mathrm{CH}_{2} \mathrm{~S}$ ), $3.86\left(\mathrm{dd}, 1 \mathrm{H}, J=10.3\right.$ and $5.4 \mathrm{~Hz}, \mathrm{CH}_{2} \mathrm{O}$ ), 3.60 (ddd, $1 \mathrm{H}, J=9.6,5.1$ and $2.3 \mathrm{~Hz}, \mathrm{H}-$ $\left.5^{\prime}\right), 2.13,2.11,2.09,2.05,2.00,1.97$ (6 s, $\left.24 \mathrm{H}, 8 \mathrm{x} \mathrm{Ac}\right) ;{ }^{13} \mathrm{C} \mathrm{NMR}\left(\mathrm{CDCl}_{3}, 75 \mathrm{MHz}\right): \delta 170.0$, 144.4, 123.5, 97.6 (C-1'), 81.9 (C-1"), 70.7 (C-3"), 69.6 (C-2'), 69.3 (C-5',5"), 69.2 (C-3'), 68.9 (C-2"), $66.4(\mathrm{C}-4 "), 66.2\left(\mathrm{CH}_{2} \mathrm{O}\right), 65.8\left(\mathrm{C}-4\right.$ '), 62.4, $62.3(\mathrm{C}-6 \mathrm{\prime}, 6 "), 49.8\left(\mathrm{CH}_{2} \mathrm{~N}\right), 24.8\left(\mathrm{CH}_{2} \mathrm{~S}\right)$, 20.9, 20.8, 20.7(MeCO); HMRS (FAB+) Calc for $\mathrm{C}_{33} \mathrm{H}_{45} \mathrm{~N}_{3} \mathrm{O}_{19} \mathrm{SNa}$ 842.2265. Found: 842.2261.

1-[2'-(2",3",4",6"-Tetra-O-acetyl- $\alpha-D-m a n n o p y r a n o s y l o x y)-e t h y l]-4-[N-a c e t y l-N-$ (2",3",,4",,6"'-tetra-O-acetyl- $\alpha$-D-mannopyranosyl)-aminomethyl]-1H-[1,2,3]-triazol (3c) (Table 1, entry 3). Solid: M.p. $89-91{ }^{\circ} \mathrm{C}$; $[\alpha]_{\mathrm{D}}+20^{\circ}$ ( $c$ 1, chloroform); IR (KBr): 1750, 1656, 1372, 1228, $1051 \mathrm{~cm}^{-1} ;{ }^{1} \mathrm{H}$ NMR $\left(\mathrm{CDCl}_{3}, 300 \mathrm{MHz}\right): \delta 7.64$ (s, $\left.1 \mathrm{H}, \mathrm{H}-5\right), 5.96$ (s, $\left.1 \mathrm{H}, \mathrm{H}-1^{\prime \prime \prime}\right)$, 5.52 (s, $1 \mathrm{H}, \mathrm{H}-2 " '), 5-31-5.09$ (m, $5 \mathrm{H}, \mathrm{H}-2 ", 3 ", 4 ", 3 " ', 4 " '), 4.79$ (d, $1 \mathrm{H}, J=16.5 \mathrm{~Hz}, \mathrm{CH}_{2} \mathrm{~N}$ ), $4.78(\mathrm{~s}, 1 \mathrm{H}, \mathrm{H}-1 "), 4.65\left(\mathrm{~d}, 1 \mathrm{H}, J=17.0 \mathrm{~Hz}, \mathrm{CH}_{2} \mathrm{~N}\right), 4.57\left(\mathrm{~m}, 2 \mathrm{H}, \mathrm{OCH}_{2} \mathrm{CH}_{2} \mathrm{~N}\right), 4.38(\mathrm{~d}, 1 \mathrm{H}$, $J=13.6 \mathrm{~Hz}, \mathrm{H}-6$ ), 4.27-4.10 (m, 3 H, H-5", H-6" or H-6"'), 4.06 (d, $1 \mathrm{H}, J=12.2$ and 2.2 Hz, H6" or H-6"'), 3.90 (m, 2 H, CH $2 \mathrm{O}$ ), 3.73 (m, 1 H, H-5"'), 2.14, 2.10, 2.09, 2.07, 12.00, 1.96, 1.56 (7 s, $27 \mathrm{H}, 9 \mathrm{Ac}) ;{ }^{13} \mathrm{C} \mathrm{NMR}\left(\mathrm{CDCl}_{3}, 75 \mathrm{MHz}\right): \bullet \delta$ 171.3, 169.9, 169.8, 169.7, 142.5, 124.1, 97.7, 79.6, 75.3, 71.1, 70.0, 69.1, 68.9, 66.3, 65.7, 65.5, 62.4, 62.2, 49.8, 40.1, 22.0, 20.8, 20.7, 20.5; HMRS (FAB+) Calc for $\mathrm{C}_{35} \mathrm{H}_{48} \mathrm{~N}_{4} \mathrm{O}_{20} \mathrm{Na}$ 867.2760. Found 267.2759.

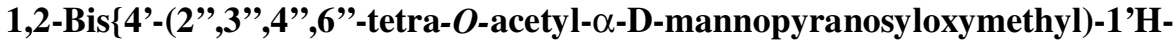

[1',2',3']-triazol-1'-yl\}-ethane (5a) (Table 1, entry 5). Solid. M.p. 59-61 ${ }^{\circ} \mathrm{C}$; $[\alpha]_{\mathrm{D}}+37^{\circ}($ c 2 , chloroform); IR (KBr) 3144, 1746, 1372, 1230, 1135, $1048 \mathrm{~cm}^{-1} ;{ }^{1} \mathrm{H} \mathrm{NMR}\left(\mathrm{CDCl}_{3}, 300 \mathrm{MHz}\right)$ : $\delta 7.43$ (s, 2 H, H-5 triazol), 5.30-5.25 (m, 4 H, H-3",4"), 5.19 (s, 2 H, H-2"), 4.96 (s, 4 H, $\left.\mathrm{CH}_{2} \mathrm{~N}\right), 4.94\left(\mathrm{~s}, 2 \mathrm{H}, \mathrm{H}-1\right.$ "), $4.80\left(\mathrm{~d}, 2 \mathrm{H}, J=12.3 \mathrm{~Hz}, \mathrm{CH}_{2} \mathrm{O}\right), 4.65(\mathrm{~d}, 2 \mathrm{H}, J=12.3 \mathrm{~Hz}$, $\mathrm{CH}_{2} \mathrm{O}$ ), 4.29 (dd, $2 \mathrm{H}, J=12.2$ and $\left.5.0 \mathrm{~Hz}, \mathrm{H}-6 "\right), 4.11$ (dd, $2 \mathrm{H}, J=12.3$ and $\left.2.4 \mathrm{~Hz}, \mathrm{H}-6 "\right)$,

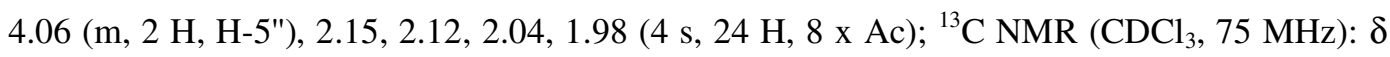
170.7, 170.1, 170.0, 169.8, 144.0, 124.3, 96.9, 69.5, 69.1, 68.8, 66.2, 62.5, 60.7, 49.6, 20.9, 20.8, 20.7, 20.7; HMRS (FAB+) Calcd. for $\mathrm{C}_{36} \mathrm{H}_{48} \mathrm{~N}_{6} \mathrm{O}_{20} \mathrm{Na}$ 907.2821. Found 907.2824.

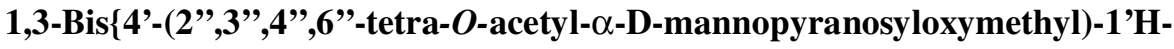

[1',2',3']-triazol-1'-methyl\}-benzene (5b) (Table 1, entry 5).. Solid. M.p. 81-83 ${ }^{\circ} \mathrm{C} ;[\alpha]_{\mathrm{D}}+16^{\circ}$ (c 1, methanol); IR (KBr): 1746, 1457, 1371, 1227, 1135, $1048 \mathrm{~cm}^{-1} ;{ }^{1} \mathrm{H}$ NMR $\left(\mathrm{CDCl}_{3}, 300\right.$ MHz): $\delta 7.55$ (s, $2 \mathrm{H}, \mathrm{H}-5$ triazol), 7.41 (t, $\left.1 \mathrm{H}, J=7.5 \mathrm{~Hz}, \mathrm{C}_{6} \mathrm{H}_{4}\right), 7.30-7.22\left(\mathrm{~m}, 3 \mathrm{H}, \mathrm{C}_{6} \mathrm{H}_{4}\right.$ ), 
5.60 (s, $4 \mathrm{H}, \mathrm{CH}_{2} \mathrm{~N}$ ), 5.31-5.26 (m, $4 \mathrm{H}, \mathrm{H}-3$ ",4"), 5.21 (br d, $2 \mathrm{H}, J=1.7 \mathrm{~Hz}, \mathrm{H}-2$ "), 4.94 (d, 2 $\mathrm{H}, J=1.7 \mathrm{~Hz}, \mathrm{H}-1$ "), 4.84 (d, $2 \mathrm{H}, J=12.4 \mathrm{~Hz}, \mathrm{CH}_{2} \mathrm{O}$ ), 4.67 (d, $2 \mathrm{H}, J=12.4 \mathrm{~Hz}, \mathrm{CH}_{2} \mathrm{O}$ ), 4.28 (dd, $2 \mathrm{H}, J=12.2$ and 5.1 Hz, H-6"), 4.12-4.00 (m, 4 H, H-5”,6”), 2.14, 2.10, 2.03, 1.99 (4 s, 24 $\mathrm{H}, 8 \mathrm{x} \mathrm{Ac}) ;{ }^{13} \mathrm{C} \mathrm{NMR}\left(\mathrm{CDCl}_{3}, 75 \mathrm{MHz}\right): \delta 170.8,170.1,169.8,144.0,135.7,130.2,128.5$, 127.6, 123.1, 97.0, 69.6, 69.1, 68.8, 66.2, 62.4, 61.2, 53.9, 20.9, 20.8, 20.7; HMRS (FAB+) Calc for $\mathrm{C}_{42} \mathrm{H}_{52} \mathrm{~N}_{6} \mathrm{O}_{20} \mathrm{Na}$ 983.3134. Found 983.3136.

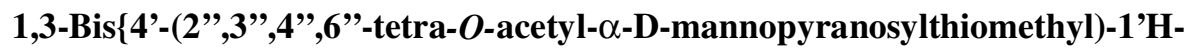
[1',2',3']-triazol-1'-methyl\}-benzene (5c) (Table 1, entry 6). Solid. M.p. 87-88 ${ }^{\circ} \mathrm{C} ;[\alpha]_{\mathrm{D}}$ $+112^{\circ}$ (c 1, chloroform); IR (KBr): 1749, 1371, 1228, $1050 \mathrm{~cm}^{-1} ;{ }^{1} \mathrm{H} \mathrm{NMR}\left(\mathrm{CDCl}_{3}, 300 \mathrm{MHz}\right)$ : $\delta 7.48$ (s, $2 \mathrm{H}, \mathrm{H}-5$ triazol), 7.25, $7.22\left(\mathrm{br} \mathrm{s}, 4 \mathrm{H}, \mathrm{C}_{6} \mathrm{H}_{4}\right), 5.51\left(\mathrm{~s}, 4 \mathrm{H}, \mathrm{CH}_{2} \mathrm{~N}\right), 5.36-5.21(\mathrm{~m}, 8 \mathrm{H}$, H-1",2",3",4"), 4.36 (ddd, $2 \mathrm{H}, J=9.8,5.0$ and $2.4 \mathrm{~Hz}, \mathrm{H}-5 "), 4.28$ (dd, $2 \mathrm{H}, J=12.1$ and 5.1, H-6"), 4.04 (dd, 2 H, $J=12.1$ and $2.1 \mathrm{~Hz}, \mathrm{H}-6 "), 3.95$ (d, $2 \mathrm{H}, J=14.6 \mathrm{~Hz}, \mathrm{CH}_{2} \mathrm{~S}$ ), 3.84 (d, $2 \mathrm{H}$, $\left.J=14.6 \mathrm{~Hz}, \mathrm{CH}_{2} \mathrm{~S}\right), 2.14,2.09,2.05,1.98(4 \mathrm{~s}, 24 \mathrm{H}, 8 \mathrm{x} \mathrm{Ac}) ;{ }^{13} \mathrm{C} \mathrm{NMR}\left(\mathrm{CDCl}_{3}, 75 \mathrm{MHz}\right): \delta$ 170.6, 169.9, 169.8, 169.6, 144.6, 135.7, 132.1, 130.0, 128.5, 127.5, 122.1, 81.7, 70.7, 69.5, 69.3, 66.2, 62.2, 53.7, 24.8, 20.8, 20.7, 20.6; HMRS (FAB+) Calc for $\mathrm{C}_{42} \mathrm{H}_{52} \mathrm{~N}_{6} \mathrm{O}_{18} \mathrm{~S}_{2} \mathrm{Na}$ 1015.2677. Found 1015.2670.

2,4,6-Tris\{2-[4'-(2",3",4”,6”-tetra-O-acetyl- $\alpha$-D-mannopyranosyloxymethyl)-1'H[1',2',3']triazol-1'-yl]-ethoxy\}-1,3,5-triazine (7a) (Table 2, entry 1).. Solid. M.p. $72-73{ }^{\circ} \mathrm{C}$; $[\alpha]_{\mathrm{D}}+32^{\circ}$ (c 1, chloroform); IR (KBr): 1755, 1561, 1234, 1134, $1048 \mathrm{~cm}^{-1} ;{ }^{1} \mathrm{H}$ NMR $\left(\mathrm{CDCl}_{3}\right.$, $300 \mathrm{MHz}$ ): $\delta 7.81$ (s, $\left.3 \mathrm{H}, \mathrm{H}-5^{\prime}\right), 5.32-5.29$ (m, 6 H, H-3",4"), 5.23 (s, 3 H, H-2"), 4.97 (s, 3 H, H-1"), 4.86-4.80 (m, $\left.15 \mathrm{H}, \mathrm{CH}_{2} \mathrm{~N}, \mathrm{CH}_{2} \mathrm{O}, \mathrm{CH}_{2} \mathrm{O}\right), 4.68$ (d, $\left.3 \mathrm{H}, J=12.2 \mathrm{~Hz}, \mathrm{CH}_{2} \mathrm{O}\right), 4.30$ (dd, 3 $\mathrm{H}, J=12.2$ and $5.0 \mathrm{~Hz}, \mathrm{H}-6 "), 4.20-4.00$ (m, $6 \mathrm{H}, \mathrm{H}-5 ", 6 "), 2.15,2.12,2.04,1.98$ (4 s, $36 \mathrm{H}$, $\mathrm{MeCO}) ;{ }^{13} \mathrm{C} \mathrm{NMR}\left(\mathrm{CDCl}_{3}, 75 \mathrm{MHz}\right): \delta 171.5$ (C-2,4,6), 170.6, 169.9, 169.8, 169.6 (CO), 142.0 (C-4'), 124.1 (C-5'), 96.9 (C-1"), 69.3 (C-2"), 69.0 (C-3"), 68.7 (C-5"), $67.0\left(\mathrm{OCH}_{2} \mathrm{CH}_{2}\right), 66.0$ (C-4"), 62.3 (C-6"), $60.8\left(\mathrm{CH}_{2} \mathrm{O}\right), 48.8\left(\mathrm{CH}_{2} \mathrm{~N}\right), 20.9,20.7,20.7,20.6$ (MeCO); HMRS (FAB+) Calc for $\mathrm{C}_{28} \mathrm{H}_{26} \mathrm{~N}_{8} \mathrm{O}_{2} \mathrm{Na}$ 529.2076. Found 529.2081.

\section{1,2,4,5-Tetrakis $\{4$ '-(2",3",4",6"-tetra-O-acetyl- $\alpha$-D-mannopyranosyloxymethyl)-} 1'H-[1',2',3']-triazol-1'-methyl)-benzene (7b) (Table 2, entry 2).. Solid. M.p. 109-111 ${ }^{\circ} \mathrm{C}$; $[\alpha]_{\mathrm{D}}+54^{\circ}$ (c 1, chloroform); IR (KBr): $v$ 3143, 1750, 1438, 1372, 1239, 1134, $1049 \mathrm{~cm}^{-1} ;{ }^{1} \mathrm{H}$ NMR $\left(\mathrm{CDCl}_{3}, 300 \mathrm{MHz}\right): \delta 7.70(\mathrm{~s}, 4 \mathrm{H}, \mathrm{H}-5$ triazol), 7.15 (s, $2 \mathrm{H}, \mathrm{Ar}), 5.68\left(\mathrm{~s}, 8 \mathrm{H}, \mathrm{CH}_{2} \mathrm{~N}\right)$, 5.35-5.25 (m, 8 H, H-3",4"), 5.20 (s, 4 H, H-2"), 4.98 (s, 4 H, H-1"), 4.83 (d, 4 H, J = 12.3 Hz, $\mathrm{CH}_{2} \mathrm{O}$ ), 4.68 (d, $4 \mathrm{H}, J=12.3 \mathrm{~Hz}, \mathrm{CH}_{2} \mathrm{O}$ ), 4.29 (dd, $4 \mathrm{H}, J=12.2$ and $\left.4.9 \mathrm{~Hz}, \mathrm{H}-6 "\right), 4.15-4.00$ (m, $8 \mathrm{H}, \mathrm{H}-5 ", 6 "), 2.14,2.11,2.04,1.98$ (4 s, $48 \mathrm{H}, 16 \mathrm{Ac}) ;{ }^{13} \mathrm{C} \mathrm{NMR}\left(\mathrm{CDCl}_{3}, 75 \mathrm{MHz}\right): \delta$ 
170.6, 169.9, 169.8, 169.6, 144.0, 134.9, 132.0, 123.6, 96.7, 69.3, 68.9, 68.6, 65.9, 62.2, 60.6, 50.5, 20.7, 20.6, 20.5; HMRS (FAB+) Calc for $\mathrm{C}_{78} \mathrm{H}_{98} \mathrm{~N}_{12} \mathrm{O}_{40} \mathrm{Na}$ 1865.5901. Found 1865.5856.

\section{1,2,3,4,5,6-Hexakis-[4-(2",3",4",6"-tetra-O-acetylø- $\alpha-D-$}

mannopyranosyloxymethyl)-1'H-[1',2',3']-1'-methyl]benzene (7c) (Table 2, entry 3).. Solid. M.p. $162-164{ }^{\circ} \mathrm{C}$; IR (KBr): 1749, 1371, 1228, 1135, 1080, $1048 \mathrm{~cm}^{-1} ;{ }^{1} \mathrm{H}$ NMR $\left(\mathrm{CDCl}_{3}, 300\right.$ MHz): $\delta 7.56(\mathrm{~s}, 1 \mathrm{H}, \mathrm{H}-5), 5.85\left(\mathrm{~s}, 2 \mathrm{H}, \mathrm{CH}_{2} \mathrm{~N}\right), 5.27$ (m, $\left.2 \mathrm{H}, \mathrm{H}-3^{\prime}, 4^{\prime}\right), 5.16$ (s, $\left.1 \mathrm{H}, \mathrm{H}-2^{\prime}\right), 4.90$ (s, $\left.1 \mathrm{H}, \mathrm{H}-1^{\prime}\right), 4.72\left(\mathrm{~d}, 1 \mathrm{H}, J=12.5 \mathrm{~Hz}, \mathrm{CH}_{2} \mathrm{O}\right), 4.59\left(\mathrm{~d}, 1 \mathrm{H}, J=12.5, \mathrm{CH}_{2} \mathrm{O}\right), 4.25(\mathrm{dd}, 1 \mathrm{H}, J$ $=11.9$ and $\left.4.1 \mathrm{~Hz}, \mathrm{H}-6^{\prime}\right), 4.05$ (m, $\left.2 \mathrm{H}, \mathrm{H}-5^{\prime}, 6^{\prime}\right), 2.13,2.10,2.05,1.98$ (4 s, $\left.12 \mathrm{H}, 4 \mathrm{x} \mathrm{Ac}\right) ;{ }^{13} \mathrm{C}$ NMR (CDCl3, 75 MHz): $\delta$ 170.8, 170.1, 170.0, 169.7, 144.2, 137.5, 123.7, 96.9, 69.5, 69.0, 68.7, 66.1, 62.3, 60.7, 48.2, 20.9, 20.8, 20.7, 20.6; HMRS (FAB+) Calc for $\mathrm{C}_{114} \mathrm{H}_{144} \mathrm{~N}_{18} \mathrm{O}_{60} \mathrm{Na}$ 2748.870. Found 2748.860.

\section{$\operatorname{Mono}\left\{\left(2^{\mathrm{I}}, 3^{\mathrm{I}}\right.\right.$-di-O-acetyl)-6'-[4'-(2",3",4",6"-tetra-O-acetyl- $\alpha-\mathrm{D}-$}

mannopyranosyloxymethyl)-1'H-[1',2',3']triazol-1-yl]-6' ${ }^{\mathrm{I}}$-deoxy $\}$ hexakis(2,3,6-tri- $O$ -

acetyl)cyclomaltoheptaose (7d) (Table 2, entry 4). Solid: M.p. $157-158^{\circ} \mathrm{C} ;[\alpha]_{\mathrm{D}}+109^{\circ}(c 1$, chloroform); IR (KBr): 1751, 1438, 1372, 1237, $1047 \mathrm{~cm}^{-1} ;{ }^{1} \mathrm{H}$ NMR $\left(\mathrm{CDCl}_{3}, 300 \mathrm{MHz}\right): \delta$ 7.70 (s, $1 \mathrm{H}, \mathrm{H}-5$ ' triazol), 5,62 (d, $1 \mathrm{H}, J=3.7 \mathrm{~Hz}, \mathrm{H}-1$ of unit I CD), 5.4-5.16 (m, $10 \mathrm{H}, \mathrm{H}-3$ of CD, H-2",3",4"), 5.14 (d, 1 H, $J=4.0$ Hz, H-1 CD), 5.1 (d, 1 H, $J=4.0$ Hz, H-1 CD), 5.08 (d, 1 $\mathrm{H}, J=4.0 \mathrm{~Hz}, \mathrm{H}-1 \mathrm{CD}), 5.07$ (d, $1 \mathrm{H}, J=4.0 \mathrm{~Hz}, \mathrm{H}-1 \mathrm{CD}), 5.03$ (d, $1 \mathrm{H}, J=3.6 \mathrm{~Hz}, \mathrm{H}-1 \mathrm{CD}$ ), 4.98 (br s, $1 \mathrm{H}, \mathrm{H}-1 "), 4.96$ (dd, $1 \mathrm{H}, J=8.6$ and $3.9 \mathrm{~Hz}, \mathrm{H}-2$ of unit I CD), 4.90-4.48, 4.364.06 (2 m, 30 H, 6 x H-2 CD, 7 x H-5 CD, 14 x H-6 CD, H-5",6",6"), 3.80-3.60 (m, 7 H, 7 x H-4 $\mathrm{CD}), 2.15-1.96(10 \mathrm{~s}, 72 \mathrm{H}, \mathrm{Ac}) ;{ }^{13} \mathrm{C} \mathrm{NMR}\left(\mathrm{CDCl}_{3}, 75 \mathrm{MHz}\right): \delta$ 170.2-169.3 (13 signals), 143.2 (C-4' triazol), 126.1(C-5' triazol), 97.1, 96.8, 96.8, 96.7, 96.6, 96.5, 96.4 (7 C-1'), 77.3, 77.2, 77.0, 76.8, 76.7, 76.4, 75.8, 71.5, 71.4, 71.2, 71.0, 70.9, 70.8, 70.4, 70.2, 70.1, 69.9, 69.6, 69.5, 69.5, 69.4, 69.3, 69.1, 68.6, 66.1, 62.7, 62.6, 62.5, 62.4, 62.3, 62.2, 60.9, 49.2, 20.8, 20.8, 20.7, 20.6; HMRS (FAB+) Calc for $\mathrm{C}_{99} \mathrm{H}_{131} \mathrm{~N}_{3} \mathrm{O}_{64} \mathrm{Na} 2509.702$. Found 2409.744.

\section{Heptakis\{2,3-di- $O$-acetyl-6-deoxy-6-[4'-(2",3",4",6"-tetra- $O$-acetyl- $\alpha$-D-} mannopyranosyloxymethyl)-1'H-[1',2',3']-triazol-1-yl]\}cyclomaltoheptaose (7e) (Table 2, entry 5). Solid. M.p. $198-200^{\circ} \mathrm{C}$; $[a]_{\mathrm{D}}+58^{\circ}$ (c 1, chloroform); IR (KBr): 1751, 1773, 1229 , $1048 \mathrm{~cm}^{-1} ;{ }^{1} \mathrm{H}$ NMR $\left(\mathrm{CDCl}_{3}, 300 \mathrm{MHz}\right): \delta 7.87$ (s, $7 \mathrm{H}, \mathrm{H}-5$ ' triazol), $5.55(\mathrm{~d}, 7 \mathrm{H}, J=3.7 \mathrm{~Hz}$, H-1), 5.41-5.18 (m, 28 H, H-3,2",3",4"), 4.98 (br s, 7 H, H-1"), 4.80 (dd, 7 H, $J=9.6$ and 3.5 $\mathrm{Hz}, \mathrm{H}-2), 4.76$ (d, $7 \mathrm{H}, J=12.5 \mathrm{~Hz}, \mathrm{CH}_{2} \mathrm{O}$ ), 4.06 (d, $7 \mathrm{H}, J=7.4 \mathrm{~Hz}, \mathrm{CH}_{2} \mathrm{O}$ ), 5.02-4.52 (m, 21 H, H-5,6,6), 4.30 (dd, 7 H, $J=12.5$ and 4.5 Hz, H-6"), 4.10 (m, 14 H, H-5",6"), 3.58 (t, 7 H, $J=$ $9.0 \mathrm{~Hz}, \mathrm{H}-4), 2.09,2.08,203,1.92,1.78$ (5 s, $126 \mathrm{H}, \mathrm{Ac}) ;{ }^{13} \mathrm{C} \mathrm{NMR}\left(\mathrm{CDCl}_{3}, 75 \mathrm{MHz}\right): \delta 170.6$, 
$170.3,169.9,169.6,169.5,143.32,126.2,96.9,96.6,67.2,69.3,69.0,68.6,65.2,62.3,60.7$, 50.2, 20.7, 20.6. HMRS (FAB+) Calc for $\mathrm{C}_{189} \mathrm{H}_{245} \mathrm{~N}_{21} \mathrm{O}_{112} \mathrm{Na} 4625.408$. Found 4625.405.

\section{Heptakis\{2,3-di-O-acetyl-6-deoxy-6-[4'-(2",3",4",6"-tetra-O-acetyl- $\alpha-D$ -} mannopyranosylthiomethyl)-1'H-[1',2',3']-triazol-1-yl]\}cyclomaltoheptaose (7f) (Table 2, entry 6).. Solid. M.p. $200-202^{\circ} \mathrm{C} ;[\alpha]_{\mathrm{D}}+122^{\circ}$ ( $c$ 1, chloroform); IR (KBr): 1751, 1229, 1049 $\mathrm{cm}^{-1} ;{ }^{1} \mathrm{H} \mathrm{NMR}\left(\mathrm{CDCl}_{3}, 300 \mathrm{MHz}\right): \delta 7.75$ (s, $7 \mathrm{H}, \mathrm{H}-5$ triazol), 5.51 (b s, $\left.7 \mathrm{H}, \mathrm{H}-1\right), 5.42$ (s, 7 H, H-1"), 5.35 (t, 7 H, J = 9.5 Hz, H-3), 5.33 (t, 7 H, J = 9.9 Hz, H-4"), 5-27 (b s, 7 H, H-2"), 5.18 (dd, 7 H, J = 9.8 and 3.0 Hz, H-3"), 4.88 (br d, 7 H, J = 14.2 Hz, H-6), 4.75 (m, 14 H, H2,6), 4.48 (m, 7 H, H-5), 4.35 (m, 7 H, H-5"), 4.31 (dd, 7 H, J = 12.1 and 4.1, H-6"), 4.35 (m, 7 H, H-5"), 4.31 (dd, 7 H, J = 12.1 and 4.1 Hz, H-6"), 4.12 (d, 7 H, J = 12.0 Hz, H-6"), 3.89 (d, 7 $\left.\mathrm{H}, \mathrm{J}=14.2 \mathrm{~Hz}, \mathrm{CH}_{2} \mathrm{~S}\right), 3.79\left(\mathrm{~d}, 7 \mathrm{H}, \mathrm{J}=14.2 \mathrm{~Hz}, \mathrm{CH}_{2} \mathrm{~S}\right.$ ), 3.54 (t, $\left.7 \mathrm{H}, \mathrm{J}=8.7 \mathrm{~Hz}, \mathrm{H}-4\right), 2.12$, 2.09, 2.05, 2.02, 1.95, 1.78 (6 s, $\left.126 \mathrm{H}, \mathrm{CH}_{3} \mathrm{CO}\right) ;{ }^{13} \mathrm{C} \mathrm{NMR}\left(\mathrm{CDCl}_{3}, 75 \mathrm{MHz}\right): \delta 170.7,170.4$, 169.9, 169.7, 144.0, 125.2, 96.7, 81.9, 76.8, 70.7, 69.7-69.3 (6 signals), 66.2, 62.3, 50.1, 24.8, 20.9, 20.8, 20.7, 20.6. HMRS (FAB+) Calc for $\mathrm{C}_{89} \mathrm{H}_{245} \mathrm{~N}_{21} \mathrm{O}_{105} \mathrm{~S}_{7} \mathrm{Na} 4738.25$ Found 4738.19.

\section{References}

(1) H. B. Mereyala, S. R. Gurrala, Carbohydr. Res. 1998, 307, 351.

(2) R. J. Kaufman, R. S. Sidhu, J. Org. Chem. 1982, 47, 4941.

(3) Z. H. Gan, R. Roy, Tetrahedron Lett. 2000, 41, 1155.

(4) A. Y. Chernyak, G. V. M. Sharma, L. O. Kononov, P. R. Krishna, A. B. Levinsky, N. K. Kochetkov, A. V. R. Rao, Carbohydr. Res. 1992, 223, 303.

(5) L. F. Zhang, Y. C. Wong, L. Chen, C. B. Ching, S. C. Ng, Tetrahedron Lett. 1999, 40, 1815.

(6) Gilbert, E. E. Patent Nº US-138, 1984 USA (CA: 106:158921).

(7) G. Van Wuytswinkel, B. Verheyde, F. Compernolle, S. Toppet, W. Dehaen, J. Chem. Soc. Perkin I 2000, 9, 1337.

(8) J. Boger, R. J. Corcoran, J. M. Lehn, Helv. Chim. Acta 1978, 6, 2190.

(9) C. RoehriStoeckel, O. Dangles, R. Brouillard, Tetrahedron Lett. 1997, 38, 1551.

(10) A. B. Theocharis, N. E. Alexandrou, A. Terzis, J. Heterocycl. Chem. 1990, 271741.

(11) J. Zavada, M. Pankova, P. Holy, M. Tichy, Synthesis 1994, 1132.

(12) W. Hayes, H. M. I. Osborn, S. D. Osborne, R. A. Rastall, B. Romagnoli, Tetrahedron Lett. 2002, 437683 . 